\title{
Thoughtless design of the electronic health record drives overuse, but purposeful design can nudge improved patient care
}

\author{
Valerie M Vaughn, ${ }^{1,2,3}$ Jeffrey A Linder ${ }^{4}$
}

${ }^{1}$ Department of Internal Medicine, University of Michigan, Ann Arbor, Michigan, USA

${ }^{2}$ Center for Clinical Management Research, VA Ann Arbor Healthcare System, Ann Arbor, Michigan, USA ${ }^{3}$ Patient Safety Enhancement Program, University of Michigan and VA Ann Arbor Healthcare System, Ann Arbor, Michigan, USA

${ }^{4}$ Department of Medicine, Division of General Internal Medicine and Geriatrics, Northwestern University Feinberg School of Medicine, Chicago, Illinois, USA

\section{Correspondence to} Dr Valerie M Vaughn, Department of Internal Medicine, University of Michigan, Ann Arbor, Ml 48109, USA; valmv@umich.edu

Accepted 27 February 2018 Published Online First 24 March 2018

\section{SLinked}

- http://dx.doi.org/10.1136/ bmjqs-2017-006899

Check for updates

To cite: Vaughn VM, Linder JA. BMJ Qual Saf 2018;27:583-586.
In his book on the psychology of persuasion, Robert Cialdini noted: "as the stimuli saturating our lives continue to grow more intricate and variable, we will have to depend increasingly on our shortcuts to handle them all". ${ }^{1}$ Such cognitive shortcuts, or heuristics, are responsible for much of human success in complex environments. Heuristics allow us to skip steps and save mental energy. Similarly, emotions provide a shorthand for remembering and reacting to complex situations and sociability allows us to function well in groups.

Medicine is the art of managing complexity. Physicians' success similarly depends on effective use of these heuristics, emotion and sociability. When admitting a patient to the hospital, a physician must diagnose the presenting symptoms, reconcile and start medications, order diagnostic testing, communicate with consultants and participate in shared-decision making. In ambulatory visits, the time pressure and tasks are slightly different, but comparably complex. In both scenarios, triaging and simultaneously managing all of the complex processes require incredible mental energy, made even more difficult by frequent interruptions. ${ }^{2}$ If every decision had to be made in an ordered, fully informed manner, we would never complete even a single patient encounter. It is no wonder we lean on heuristics and emotional and social influences in our decision-making. They often serve us well.

Despite their utility, heuristics, emotion and social influences can lead to predictably poor choices. Richard Thaler won the 2017 Nobel Prize in Economics, largely for recognising this fact. ${ }^{3}$ Thaler also described a process, termed 'nudging', by which environments are re-designed to take advantage of heuristics to encourage better choices. ${ }^{4}$ Thaler's work focuses on public policy and public health, but the same concepts of environment influencing behaviour is seen in the interaction between physician decision-making and the design of the electronic health record (EHR).

For better or worse, the EHR has become an integral part of medical care. For every hour we spend on direct patient care, we spend another two with the EHR. ${ }^{5}$ Even when interacting with patients, our focus is on computer screens up to $80 \%$ of the time. ${ }^{6}$

Given this degree of attention, it is not surprising that the EHR influences physician behaviour, especially the overuse of low-value medical care. For example, an unchecked box on an order set provides a powerful stimulus to order a test, regardless of clinical utility. ${ }^{7}$ Displaying brand name instead of generic options leads to more expensive prescribing. ${ }^{8}$ Allowing labs to be ordered recurrently increases unnecessary phlebotomy. ${ }^{9}$ Even individually listing inappropriate antibiotics (rather than grouping them) can make them more noticeable, resulting in more broad-spectrum use. ${ }^{10}$

Despite the known effect EHR has on our choices, how often is it purposefully designed? Presently, when a new order set is created, is influence on clinician behaviour considered? Which options are listed for testing and treatment? All options? Or just clinically appropriate ones? How are they listed? Alphabetically? Numerically? Or are recommended and less-expensive options listed first? 
Consider a poorly designed EHR order set you may have previously encountered for a common diagnosis such as community-acquired pneumonia. In it, antibiotics are listed alphabetically (making amikacin the first choice) and 'morning labs' are automatically ordered ad infinitum. Large lists contain all possible diagnostic tests (even costly tests for rare diseases) with extremely expensive tests listed before cheaper alternatives. When an antibiotic is selected, the route is automatically intravenous even for stable patients tolerating oral medications. Through its poor design, this order set becomes a hindrance to appropriate decision-making and can amplify overuse and overspending.

With a little more planning, we could combat reflexive overuse by repurposing the EHR to improve decision-making. Take for example the problem of antibiotic overuse, which over the last half century has contributed to the rise of antibiotic-resistant bacteria and Clostridium difficile and threatened to propel us into a post-antibiotic era. ${ }^{11}$ Despite knowledge of the problem, rampant antibiotic overuse has continued-in part due to the complexity of diagnosing infections, fear of complications and a belief that patients want antibiotics. Together, these cognitive, emotional and social pressures lead to shortcuts that often trigger antibiotic overuse. Fortunately, there have been broad strides in improving antibiotic use through an array of interventions using what we have learnt about choices from behavioural economics and social psychology.

One of the most powerful nudges for changing behaviour is using defaults or reframing the status quo. The status quo bias is a strong driver of behaviour: people tend to stay on the path in motion or repeat previously made decisions. Operational examples include having people 'opt out' rather than 'opt in', using automatic enrolment, or by highlighting the default when providing choices. ${ }^{12-14}$ Importantly, default-based nudges allow those with true reservations to 'opt out', maintaining physician autonomy if patient conditions are unusual. However, those without well-formed opinions (the majority) will typically choose the default. If the default is purposeful, this can lead to improved care and reduced overuse. ${ }^{8121315}$

Recently, in BMJ Quality and Safety, Munigala et $a l^{16}$ demonstrated the powerful effect of changing the default to reduce urine culture ordering. Initially, their emergency department listed urinalysis with reflex to urine culture in commonly ordered tests-in essence, setting this as the default urine test. The authors' intervention was simple. They removed reflex culture from this order set. Thus, the remaining test (urinalysis with reflex to microscopy) became the new default and daily urine culture rates were cut in half. Furthermore, since there was no increase in urine culture testing in the first 24 hours after hospitalisation, physicians who had strong clinical reasons to order urine cultures in the emergency department (eg, due to patient symptoms) likely did. Although the investigators did not have antibiotic prescribing data, given what we also know about how positive urine cultures trigger antibiotic use, this simple intervention has the potential to reduce antibiotic overuse.

Beyond defaults, the EHR also has potential to reduce overuse by altering the emotional overreaction we clinicians often have to positive diagnostic results. Generally, positive results induce a knee-jerk reaction to act. This reflex is emotionally laden. Physicians fear 'missing something' far more than they fear a side effect of a well-intentioned treatment. ${ }^{17}$ Instead, they imagine the judgement of their colleagues at the next morbidity and mortality if they did not treat a 'positive' culture-only to have the patient suffer a horrible outcome. That extreme (though unlikely) possibility exerts far more influence than the more likely, but less striking, risk of an antibiotic-related adverse reaction.

The EHR can reduce reflexive treatment through 'diagnostic stewardship'. ${ }^{18}$ For example, in one powerful intervention, all urine culture results (except those in catheterised patients) were hidden from clinicians' view in the EHR. When results were positive, a message was displayed explaining that most positive urine cultures represent asymptomatic bacteriuria and asking clinicians to call for results if they strongly suspected a urinary tract infection. Treatment of asymptomatic bacteriuria fell by $75 \%{ }^{19}$ A similar approach of hiding results and providing thoughtful messaging through the EHR could be applied to many overused diagnostic tests, especially those with high rates of false-positive results.

Emotional triggers can be used to change behaviour in other ways. For example, a monthly antibiotic prescribing report, comparing clinicians to their peers, telling most they were 'not top performers', appeals to our strong internal desire to be competent and galvanises clinicians to improve their performance. In one study, such messages decreased inappropriate antibiotic prescribing from $20 \%$ to $4 \%$ and had persistent effects 12 months after the intervention was turned off. ${ }^{20} 21$ Although the intervention itself was 'outside' the EHR, this emotionally laden intervention powerfully affected EHR-based antibiotic ordering.

Finally, the EHR can capitalise on social and cultural norms that guide behaviour. By understanding and shaping clinician norms, we can influence their behaviour. Although some EHRs require an indication for ordering antibiotics, the rationale for antibiotic prescribing is often not explicit. If, however, EHRs require a justification that is automatically included in the patient's medical record as an 'antibiotic justification note', it becomes explicit 
and public. Having their decision-making exposed in this way, physicians decreased inappropriate antibiotic prescribing from $23 \%$ to $5 \% .^{2122}$

Using what we know about nudges, imagine what a poorly designed order set would look like if it were thoughtfully designed. The first antibiotic listed would be the antibiotic recommended by local guidelines (in large font and individually). The computer would automatically check renal function, clinical stability and ability to swallow pills before recommending an initial dose and route of administration. The list of diagnostic tests would show the most appropriate, common and affordable options first, and would alert the user if a test were ordered which had already recently resulted. The urine culture's positive result would be suppressed and instead describe symptoms that would qualify for treatment. When a clinician decides to prescribe a fluoroquinolone antibiotic (despite institutional recommendations against), they would be informed they are not a 'top performer' compared with their peers and asked to justify their choice (which would be automatically entered into the medical record). Each element of such an order set is now purposefully designed to influence clinicians to make appropriate treatment decisions. In doing so, overuse is reduced, patient care is improved and decision-making is made easier by using-rather than fighting-clinicians' inherent skills.

As alternatives become more numerous and more complex, choice architects have more to think about and more work to do, and are much more likely to influence choices (for better or for worse).- - Richard $\mathrm{H}$ Thaler, $\mathrm{PhD}^{4}$

\section{CONCLUSION}

A critical first step in improving clinician behaviour is recognising that most decisions occur with little active deliberation. When making rapid choices, clinicians are being influenced by EHR design, defaults, diagnostic stimuli, emotion and social norms-whether that influence is purposeful or not. To improve, we must recognise these tendencies and use thoughtful design to capitalise on the powerful potential of the EHR to nudge our behaviour towards better patient care.

\section{Twitter@ValerieVaughnMD@jeffreylinder}

Funding The authors have not declared a specific grant for this research from any funding agency in the public, commercial or not-for-profit sectors.

Competing interests None declared.

Patient consent Not required.

Provenance and peer review Commissioned; internally peer reviewed.

(C) Article author(s) (or their employer(s) unless otherwise stated in the text of the article) 2018. All rights reserved. No commercial use is permitted unless otherwise expressly granted.

\section{REFERENCES}

1 Cialdini RB. Influence: the psychology of persuasion. New York, NY: HarperBusiness, 2006 (first published 1984).

2 Westbrook JI, Raban MZ, Walter SR, et al. Task errors by emergency physicians are associated with interruptions, multitasking, fatigue and working memory capacity: a prospective, direct observation study. BMJ Qual Saf 2018;27:655-63.

3 The Prize in Economic Science 2017-Press Release [Web]. Nobelprize.org: Nobel Meida AB. 2017. http://www. nobelprize.org/nobel_prizes/economic-sciences/laureates/2017/ press.html (accessed 19 Jan 2017).

4 Thaler Richard H, Sunstein Cass R. Nudge: improving decisions about health, wealth, and happiness. New Haven, CT: Yale University Press, 2008.

5 Sinsky C, Colligan L, Li L, et al. Allocation of physician time in ambulatory practice: a time and motion study in 4 specialties. Ann Intern Med 2016;165:753-60.

6 Frankel RM. When it comes to the physician-patientcomputer relationship, the "eyes" have it. Distracted Doctoring: Springer, 2017:87-99.

7 Leis B, Frost A, Bryce R, et al. Standard admission order sets promote ordering of unnecessary investigations: a quasirandomised evaluation in a simulated setting. BMJ Qual Saf 2017;26:938-40.

8 Patel MS, Day S, Small DS, et al. Using default options within the electronic health record to increase the prescribing of generic-equivalent medications: a quasi-experimental study. Ann Intern Med 2014;161(10 Suppl):S44-S52.

9 Pageler NM, Franzon D, Longhurst CA, et al. Embedding time-limited laboratory orders within computerized provider order entry reduces laboratory utilization. Pediatr Crit Care Med 2013;14:413-9.

10 Tannenbaum D, Doctor JN, Persell SD, et al. Nudging physician prescription decisions by partitioning the order set: results of a vignette-based study. J Gen Intern Med 2015;30:298-304.

11 CDC. Antibiotic resistance threats in the United States, 2013.

12 Halpern SD, Loewenstein G, Volpp KG, et al. Default options in advance directives influence how patients set goals for endof-life care. Health Aff 2013;32:408-17.

13 Johnson EJ, Goldstein D. Do defaults save lives? Science 2003;302:1338-9.

14 Madrian BC, Shea DF. The power of suggestion: inertia in 401(k) participation and savings behavior. Q J Econ 2001;116:1149-87.

15 Halpern SD, Ubel PA, Asch DA. Harnessing the power of default options to improve health care. N Engl J Med 2007;357:1340-4.

16 Munigala S, Jackups RR, Poirier RF, et al. Impact of order set design on urine culturing practices at an academic medical centre emergency department. BMJ Qual Saf 2018;27:587-92.

17 Livorsi D, Comer A, Matthias MS, et al. Factors influencing antibiotic-prescribing decisions among inpatient physicians: a qualitative investigation. Infect Control Hosp Epidemiol 2015;36:1065-72.

18 Morgan DJ, Malani P, Diekema DJ. Diagnostic stewardshipleveraging the laboratory to improve antimicrobial use. JAMA 2017;318:607-8.

19 Leis JA, Rebick GW, Daneman N, et al. Reducing antimicrobial therapy for asymptomatic bacteriuria among noncatheterized inpatients: a proof-of-concept study. Clin Infect Dis 2014;58:980-3. 


\section{Editorial}

20 Linder JA, Meeker D, Fox CR, et al. Effects of behavioral interventions on inappropriate antibiotic prescribing in primary care 12 months after stopping interventions. JAMA 2017;318:1391-2.

21 Meeker D, Linder JA, Fox CR, et al. Effect of behavioral interventions on inappropriate antibiotic prescribing among

primary care practices: a randomized clinical trial. JAMA 2016;315:562-70.

22 Persell SD, Doctor JN, Friedberg MW, et al. Behavioral interventions to reduce inappropriate antibiotic prescribing: a randomized pilot trial. BMC Infect Dis 2016;16:373. 